\title{
Temperature, water activity and gas composition effects on the growth and aflatoxin production by Aspergillus flavus on paddy
}

\begin{abstract}
The main aim of this study was to evaluate the combined effects of temperature with water activity (aw) and $\mathrm{CO} 2$ with aw on the growth and aflatoxin production by Aspergillus flavus Link on paddy. The effects of temperature $\left(20-30^{\circ} \mathrm{C}\right)$ and aw $(0.92-0.98)$ on the relationship between colony diameter and aflatoxin production, and the influence of aw (0.92-0.98) and $\mathrm{CO} 2(20-80 \%)$ on the growth and toxin production were studied using full factorial design. Colony diameters were regularly measured and aflatoxins were periodically analyzed using HPLC with fluorescence detector. The growth and aflatoxin formation increased with aw at the temperatures studied, and toxin production was positively correlated with the incubation time and colony diameter. Except at 0.92 aw, as much as $80 \% \mathrm{CO} 2$ failed to inhibit the growth of fungi completely. However, at all aw levels studied the growth parameters as estimated by Baranyi function and aflatoxin were affected by the increment in $\mathrm{CO} 2$ where growth rates and aflatoxin were negatively correlated with $\mathrm{CO} 2$ while the lag phase durations were positively correlated with CO2. Under 0.98 aw, the atmosphere enriched with $20 \%$ and $80 \% \mathrm{CO} 2$ lead to at least $59 \%$ and $88 \%$ reduction in growth and $47 \%$ and $97 \%$ in the toxin production, respectively. At $0.95 \mathrm{aw}$, the lag phases of both isolates in average increased by a factor of 1.7-12 when the CO2 levels in the headspace were between 20 and $80 \%$ compared to the control. The growth rate and lag phase durations under the modified atmospheres were successfully described using a polynomial equation $(\mathrm{R} 2>0.97)$. The results of the study could form a basis of indicative guidelines on the possible control of A. flavus and aflatoxin in paddy during temporary storage prior to drying.
\end{abstract}

Keyword: Aspergillus flavus; Modified atmospheres; Water activity; Temperature; Aflatoxin; Growth; Paddy 